\title{
Advanced Control Design and Fault Diagnosis
}

\author{
Silvio Simani ${ }^{1, *}$ (1) and Elena Zattoni ${ }^{2}$ (1) \\ 1 Engineering Department, University of Ferrara, Via Saragat 1E, 44123 Ferrara, Italy \\ 2 Dipartimento di Ingegneria dell'Energia Elettrica e dell'Informazione "G. Marconi", Alma Mater Studiorum \\ Università di Bologna, Viale Risorgimento 2, 40136 Bologna, Italy; elena.zattoni@unibo.it \\ * Correspondence: silvio.simani@unife.it
}

check for updates

Citation: Simani, S.; Zattoni, E. Advanced Control Design and Fault Diagnosis. Energies 2021, 14, 5699. https://doi.org/10.3390/en14185699

Received: 3 September 2021

Accepted: 7 September 2021

Published: 10 September 2021

Publisher's Note: MDPI stays neutral with regard to jurisdictional claims in published maps and institutional affiliations.

Copyright: (c) 2021 by the authors. Licensee MDPI, Basel, Switzerland. This article is an open access article distributed under the terms and conditions of the Creative Commons Attribution (CC BY) license (https:/ / creativecommons.org/licenses/by/ $4.0 /)$.

\begin{abstract}
This document provides the motivations and a brief introduction to the Special Issue entitled "Advanced Control Design and Fault Diagnosis", which aims at presenting several solutions to the advanced control design and fault diagnosis systems. These methodologies can be considered in the general framework of advanced control, fault diagnosis and fault tolerant control systems, which are also able to improve the safety of the system under monitoring. The focuses of the current research in this field addressed in this Special Issue are also presented with emphasis on the practical application to simulated and realistic examples, which should provide an overall picture of current and future developments in this area. The works of this Special Issue represent suitably extended contributions selected by the proponents from the ACD2019-the 15th European Workshop on Advanced Control and Diagnosis, which was organised in Bologna, Italy on 21st-22nd November.
\end{abstract}

Keywords: advanced control; fault diagnosis; fault tolerant control; reliability and robustness; safety-critical systems

\section{Introduction}

Modern technological and safety-critical systems rely on sophisticated control solutions to meet increased performance in faulty conditions, reliability and safety requirements. A conventional feedback control design for a complex system may result in an unsatisfactory performance, or even instability, in the event of malfunctions in actuators, sensors or other system components. To overcome this limitation, new approaches to advanced control system design and fault diagnosis strategies have been developed in order to tolerate component malfunctions while maintaining the desirable stability and performance properties. This feature is particularly important for safety-critical systems. In such plants, the consequences of a minor (abrupt or incipient) fault in a system component can be catastrophic. Therefore, the demand on reliability, safety, availability and fault tolerance is generally high. It is necessary to design control strategies that are capable of tolerating potential faults in order to improve the reliability, safety and availability, while providing desirable performances. These types of control systems are known as fault tolerant control systems. In more detail, they consist of control systems possessing the ability to accommodate component faults automatically. They are also capable of maintaining overall system stability and acceptable performance in the event of such faults. In other words, a closed-loop control system that can tolerate component malfunctions, while maintaining desirable performance and stability properties is considered to be a fault tolerant control system [1].

On the other hand, over the last four decades, the growing demand for safety, reliability, maintainability and survivability in technical systems has drawn significant research in fault detection and diagnosis, resulting in the development of many solutions. Note that in the literature, fault detection and isolation or fault detection and identification (estimation) are often used. In advanced control design for fault diagnosis, fault identification (i.e., estimation) is important; therefore, the fault detection and diagnosis tasks are mainly 
considered to highlight the requirement of fault reconstruction. On a parallel path, research on reconfigurable fault tolerant control systems has increased progressively since the initial research on restructurable control and self-repairing flight control systems began in the early 1970s. More recently, fault tolerant control has attracted more and more attention in both industry and academic communities due to the increased demands for safety, high system performance, productivity and operating efficiency in a wider range of engineering applications, not limited to traditional safety-critical systems [2,3].

From the beginning of 1980, several milestones have been available in the literature. In particular, apart from the triennial IFAC Symposium on Fault Detection, Supervision and Safety for Technical Process (SAFEPROCESS) started in 1991, in 2010, the first International Conference on Control and Fault Tolerant Systems (Systol'10) was a success and demonstrated the demand for establishing a permanent scientific forum in the general area of system monitoring, fault diagnosis and fault tolerant control. The second conference on Control and Fault Tolerant Systems (Systol'13), through its technical program, provided a unique opportunity for the academic and industrial community to formulate new challenges, share solutions and discuss future research directions. Presentations of theoretical results accompanied by practice-related experiments were encouraged, and the best contributions were selected for a Special Issue from the latest Systol'16 Conference held in Barcelona, Spain, 7-9 September 2016.

On the other hand, regarding the Advanced Control and Diagnosis (ACD) workshops, the first one was organised in Duisburg, Germany, in November 2003. After six editions, the ACD workshop became a European event, when it was organised in Ferrara, Italy, in 18-19 November 2010. After six further editions, the ACD2019 became an intercontinental event, with its edition in Bologna, Italy, 21-22 November 2019.

Historically, from the point of view of practical applications, a significant amount of research on advanced control design and fault diagnosis systems was motivated by aircraft flight control system design. The key point was to provide fault accommodation features to ensure a safe flight in the event of severe faults in the aircraft. Such effort was also stimulated partly by two commercial aircraft accidents in the late 1970s, in particular involving Delta Flight 1080 (12 April 1977), and American Airlines DC-10, in Chicago. It is, thus, evident why the fault tolerant control problem began to draw more and more attention in a wider range of academic and application communities, due to increased safety and reliability demands beyond what a conventional control system can offer [4].

This Special Issue highlights that, maybe due to historical reasons and the complexity of the problem, most of the research on advanced control design and fault diagnosis was carried out as two separate tasks. More specifically, most of the fault diagnosis techniques are developed as a diagnostic or monitoring tool, rather than an integral part of the advanced control design. As a result, some existing fault detection methods may not satisfy the requirements of advanced control solutions. On the other hand, most of the research on advanced control design is carried out assuming the availability of a perfect fault diagnosis. Little attention has been paid to the analysis and design with the overall system structure and interaction between advanced control and fault diagnosis. From the viewpoint of control design, what are the needs and requirements for fault diagnosis? How can the advanced control and fault diagnosis be designed in an integrated manner for safety-critical applications? Many challenging topics, which are analysed by the contributions to this Special Issue, properly selected from the ACD2019 workshop (https:/ / eventi.unibo.it/acd2019/, accessed on 31 August 2021), still remain open for further research and development $[2,3]$.

One of the motivations of this Special Issue is to provide an overview on the recent developments in advanced control design and fault diagnosis, with applications to realistic systems. At the same time, this Special Issue aims at presenting some challenging open problems for future research. It is the Editors' hope that this Special Issue could provide some useful information to researchers and practitioners in the field in order to facilitate the further development of this important area. 


\section{Contributions' Description}

The papers contributed to the Special Issue were selected from the best contributions presented during the ACD2019-15th European Workshop on Advanced Control and Diagnosis, Alma Mater Studiorum - University of Bologna that was held in Bologna, Italy, on 21-22 November 2019, and they are given here in an extended version with in-depth analysis and new contributions. Their details and a brief summary of the addressed issues have been provided in the following.

- Remaining Useful Life Prediction of MOSFETs via the Takagi-Sugeno Framework by Marcin Witczak, Marcin Mrugalski and Bogdan Lipiec [5].

The work develops a new method for predicting the remaining useful life of technical devices, which relies on a soft computing approach in order to bridge the gap between analytical and data-driven health prognostic methods. Therefore, a practical method for calculating the remaining useful life of electronic components is addressed. Differently from the approaches available in the related literature, the proposed strategy also provides the uncertainty interval containing an expected remaining useful life.

- The Short-Circuit Protections in Hybrid Systems with Low-Power Synchronous Generators by Bartosz Rozegnał, Paweł Albrechtowicz, Dominik Mamcarz, Natalia RadwanPragłowska and Artur Cebula [6].

The work considers the case of single-phase short-circuits, as they represent the most common faults in electrical systems. In particular, backup power supply systems from small power synchronous generators are considered. Moreover, standard protection systems for these devices work correctly during supply from the grid, but do not guarantee fast off-switching, while short-circuits occur during supplication from the backup generator set. Therefore, an accurate analysis is performed to highlight the main problems and the adopted solutions.

- Model Predictive Control for Virtual Synchronous Generator with Improved Vector Selection and Reconstructed Current by Nan Jin, Chao Pan, Yanyan Li, Shiyang Hu and Jie Fang [7].

The paper considers the problem of the degradation of the inertia and the stability features of power grids due to the large-scale renewable energy conversion systems. Therefore, in order to improve the grid recovery, this study develops a model predictive control scheme that can automatically control the generated output power with the grid frequency and voltage changes. The fault tolerant ability and the achieved reliability characteristics are also investigated by means of experimental investigations.

- Model Based Optimisation Algorithm for Maximum Power Point Tracking in Photovoltaic Panels by Faiçal Hamidi, Severus Constantin Olteanu, Dumitru Popescu, Houssem Jerbi, Ingrid Dincă, Sondess Ben Aoun and Rabeh Abbassi [8].

This study proposes an enhanced maximisation problem based on gradient optimisation techniques to achieve better performance. This issue is considered in the context of maximum power point tracking for photovoltaic panels, where an equality restriction applies, which is solved by employing advanced optimisation and minimisation tools. The simulation of a basic conversion system was considered to validate the performances of the developed tools.

- Testing System for the On-Site Checking of Magneto-Thermal Switches with Arc Fault Detection by Giovanni Bucci, Fabrizio Ciancetta, Andrea Fioravanti, Edoardo Fiorucci, Simone Mari and Alberto Prudenzi [9].

This work addresses an experimental test method to verify the proper functioning of magneto-thermal switches with an electric arc protection function for the occurrence of arc faults. In fact, arcing represents a harmful condition that can lead to electrical fires, 
especially if occurring in residential areas. The achieved results show that the proposed method adequately detected the correct operations and tripping time by using different surge suppressors. The proposed system lays the foundations for a portable test system for the periodic checking of electrical systems in which arc fault detection devices are installed.

- Density Difference Grid Design in a Point-Mass Filter by Jakub Matoušek, Jindřich Duník and Ondřej Straka [10].

The paper deals with the Bayesian state estimation of nonlinear stochastic dynamic systems. In particular, it is focused on the point-mass filter, solving the Bayesian recursive relations for the state estimate conditional density computation using the deterministic grid-based numerical integration method. A simulation example is considered to validate the characteristics of the proposed grid design.

- An Efficient Robust Predictive Control of Main Steam Temperature of Coal-Fired Power Plant by Di Wang, Xiao Wu and Jiong Shen [11].

This work develops an offset-free offline robust model predictive control strategy for the main steam temperature system of a coal-fired power plant. This issue represents the key point as it determines the economic efficiency and safety of the process. A zonotopetype uncertain model is exploited as the prediction model in the proposed design due to its features of high accuracy, compactness of representation and reduced complexity. An offline algorithm aiming at the system robustness and computational efficiency is then developed to maintain the desired steam temperature in case of wide operating condition changes. Simulations using on-site operating data of a $1000 \mathrm{MW}$ ultra-supercritical power plant serve to highlight the satisfactory control performance and online computation efficiency.

- Advanced Control for Hydrogen Pyrolysis Installations by Dumitru Popescu, Catalin Dimon, Pierre Borne, Severus Constantin Olteanu and Mihaela Ancuta Mone [12].

This paper aims at designing a numerical control solution based on the thermodynamic analysis of the pyrolysis reactions for hydrogen production and to present novel research developments that highlight industrial applications. In fact, today, hydrogen production plays an important part in the industry due to the increasing use of hydrogen in significant domains, such as chemistry, transportation, or energy. Therefore, the study considers the thermodynamic evaluation of the system equilibrium for the pyrolysis reactions set, to recommend an appropriate automatic control solution for hydrogen pyrolysis installations. The data from an experimental platform were employed to validate the achieved results.

- Doubly Fed Induction Generator Open Stator Synchronised Control during Unbalanced Grid Voltage Condition by Akrama Khan, Xiao Ming Hu, Mohamed Azeem Khan and Paul Barendse [13].

The paper proposes a systematic synchronisation procedure for a doubly fed induction generator during unbalanced grid voltage conditions. The initial induced voltage at the open stator terminal is required to synchronise with the grid voltage in magnitude, frequency and phase. An open stator negative sequence rotor current controller is implemented with the conventional doubly fed induction generator vector controller, which allows the induced stator voltage to become as unbalanced as the grid voltage, hence enabling a smooth connection. The procedure is validated experimentally on a $2.2 \mathrm{~kW}$ laboratory-scaled test bench.

\section{Conclusions}

The major objective of this Special Issue was to introduce and revise design methods for advanced control and fault diagnosis for dynamic processes from different aspects. Under the view of system types, advanced control design and fault diagnosis issues were dealt with for linear time-invariant and time-varying systems as well as for nonlinear and complex systems. From the methodological point of view, both model-based and data-driven schemes were investigated. To allow for a self-contained study and enable an 
easy implementation in realistic and engineering applications, the necessary knowledge as well as tools in mathematics and control theory were considered in this Special Issue. The main results with the advanced control design and fault diagnosis schemes were presented in the form of algorithms and demonstrated also by means of benchmark case studies. The intended audience of this Special Issue are process and control engineers, engineering students and researchers with a control engineering background.

Funding: This research received no external funding.

Institutional Review Board Statement: Not applicable.

Informed Consent Statement: Not applicable.

Data Availability Statement: Not applicable.

Conflicts of Interest: The authors declare no conflict of interest.

\section{References}

1. Blanke, M.; Kinnaert, J.; Lunze, J.; Staroswiecki, M.; Schroder, J. Diagnosis and Fault-Tolerant Control, 1st ed.; Springer: Berlin, Germany, 2003; ISBN 3540010564.

2. Chen, J.; Patton, R.J. Robust Model-Based Fault Diagnosis for Dynamic Systems; Kluwer Academic Publishers: Dordrecht, The Netherlands, 1999.

3. Ding, S.X. Model-Based Fault Diagnosis Techniques: Design Schemes, Algorithms, and Tools, 1st ed.; Springer: Berlin/Heidelberg, Germany, 2008; ISBN 978-3540763031.

4. Stevens, B.L.; Lewis, F.L. Aircraft Control and Simulation, 2nd ed.; John Wiley and Sons: Hoboken, NJ, USA, 2003.

5. Witczak, M.; Mrugalski, M.; Lipiec, B. Remaining Useful Life Prediction of MOSFETs via the Takagi-Sugeno Framework. Energies 2021, 14, 2135. [CrossRef]

6. Rozegnał, B.; Albrechtowicz, P.; Mamcarz, D.; Radwan-Pragłowska, N.; Cebula, A. The Short-Circuit Protections in Hybrid Systems with Low-Power Synchronous Generators. Energies 2021, 14, 160. [CrossRef]

7. Jin, N.; Pan, C.; Li, Y.; Hu, S.; Fang, J. Model Predictive Control for Virtual Synchronous Generator with Improved Vector Selection and Reconstructed Current. Energies 2020, 13, 5435. [CrossRef]

8. Hamidi, F.; Olteanu, S.C.; Popescu, D.; Jerbi, H.; Dincă, I.; Ben Aoun, S.; Abbassi, R. Model Based Optimisation Algorithm for Maximum Power Point Tracking in Photovoltaic Panels. Energies 2020, 13, 4798. [CrossRef]

9. Bucci, G.; Ciancetta, F.; Fioravanti, A.; Fiorucci, E.; Mari, S.; Prudenzi, A. Testing System for the On-Site Checking of MagnetoThermal Switches with Arc Fault Detection. Energies 2020, 13, 4652. [CrossRef]

10. Matoušek, J.; Duník, J.; Straka, O. Density Difference Grid Design in a Point-Mass Filter. Energies 2020, 13, 4080. [CrossRef]

11. Wang, D.; Wu, X.; Shen, J. An Efficient Robust Predictive Control of Main Steam Temperature of Coal-Fired Power Plant. Energies 2020, 13, 3775. [CrossRef]

12. Popescu, D.; Dimon, C.; Borne, P.; Olteanu, S.C.; Mone, M.A. Advanced Control for Hydrogen Pyrolysis Installations. Energies 2020, 13, 3270. [CrossRef]

13. Khan, A.; Hu, X.M.; Khan, M.A.; Barendse, P. Doubly Fed Induction Generator Open Stator Synchronized Control during Unbalanced Grid Voltage Condition. Energies 2020, 13, 3155. [CrossRef]

\section{Short Biography of Authors}

S. Simani was born in Ferrara in 1971. He received his Laurea degree (cum laude) in Electronic Engineering from the Department of Engineering at the University of Ferrara, Italy, in 1996, and was awarded a Ph.D. in Information Science (Automatic Control) at the Department of Engineering of the University of Ferrara and Modena, Italy, in 2000. Since February 2002, he has been an Assistant Professor at the Department of Engineering of the University of Ferrara, and since December 2018, he has been a Professor of Automatic Control at the same Department. Since 1999, he has been a member of the IFAC Technical Committee 6.4 on Fault Detection, Supervision and Safety for Technical Processes (SAFEPROCESS), and vice-chair of the same TC from 2018. Since 1998, he has been a member of the IEEE Society, and since 2016, he has been a Senior Member IEEE. Prof. Simani has published more than 260 refereed journal and conference papers, several book chapters and four monographs. His research interests include fault diagnosis and fault tolerant control of linear and nonlinear dynamic processes, system modelling, identification and data analysis, linear and nonlinear filtering techniques, fuzzy logic and neural networks for modelling and control, as well as the interaction issues among identification, fault diagnosis, fault tolerant and sustainable control. These techniques have been applied to power plants, renewable energy conversion systems, aircraft and spacecraft processes.

E. Zattoni received a Laurea degree with Honours in Electronics Engineering in 1995 and a Ph.D. in Systems Engineering in 1999 from the University of Bologna, Italy. She is an Associate Professor of Automatic Control at the Alma Mater Studiorum Università di Bologna. She has been a Visiting Professor at Leuphana University, Aalto University, University of Michigan, Institut de Recherche en Communications et Cybernétique de Nantes, and Brown University. Her research interests are focused on the geometric and 
structural approaches to control systems, including hybrid systems and time-delay systems, with applications to the synthesis of enhanced-reliability control systems. She has authored more than one hundred and fifty papers in the field. She has co-edited the book "Structural Methods in the Study of Complex Systems". She has been the General Chair of the "15 ${ }^{\text {th }}$ European Workshop on Advanced Control and Diagnosis". She is a member of the IFAC Technical Committee 2.2 on "Linear Control Systems" and coordinator of the TC2.2 Working Group on "Control of Complex Systems". She is the General Chair of the "1st IFAC Workshop on Control of Complex Systems" (COSY 2022), to be held in Bologna in 2022, and IPC Co-Chair of the "8th IFAC Symposium on System Structure and Control" (SSSC 2022), to be held in Montreal in 2022. She is a Senior Member of IEEE, and she has served on the Member Activity Board of the IEEE Control Systems Society. 